\title{
Video Painting with Space-Time-Varying Style Parameters
}

\author{
Mizuki Kagaya, William Brendel, Qingqing Deng, Todd Kesterson, Sinisa Todorovic, Patrick J. Neill, \\ and Eugene Zhang, Member, IEEE
}

\begin{abstract}
Artists use different means of stylization to control the focus on different objects in the scene. This allows them to portray complex meaning and achieve certain artistic effects. Most prior work on painterly rendering of videos, however, uses only a single painting style, with fixed global parameters, irrespective of objects and their layout in the images. This often leads to inadequate artistic control. Moreover, brush stroke orientation is typically assumed to follow an everywhere continuous directional field. In this article, we propose a video painting system that accounts for the spatial support of objects in the images or video, and uses this information to specify style parameters and stroke orientation for painterly rendering. Since objects occupy distinct image locations and move relatively smoothly from one video frame to another, our object-based painterly rendering approach is characterized by style parameters that coherently vary in space and time. Space-time-varying style parameters enable more artistic freedom, such as emphasis/deemphasis, increase or decrease of contrast, exaggeration or abstraction of different objects in the scene in a temporally coherent fashion.
\end{abstract}

Index Terms-Non-photorealistic rendering, video painting, multi-style painting, tensor field design

\section{INTRODUCTION}

$\mathrm{P}$ AINTERLY rendering of images and videos has received much attention in the past two decades. In this article, we describe a video painting framework in which style parameters as well as brush stroke orientations can be specified individually for each region (object or background) in some keyframes and propagated to other frames in a temporally coherent fashion. We will refer to this as the problem of multistyle painterly rendering, or simply multi-style painting. There are a number of benefits in multi-style painting:

1) By spatially varying style parameters such as brush stroke length, width, and opacity, the artist has the freedom to emphasize or deemphasize certain objects in the scene (Fig. 1 (c): flower (emphasized) and leaves (deemphasized)), to control the level of abstraction or realism in the resulting painting (Fig. 2 (d-e)), to increase or decrease contrasts between neighboring objects (Fig. 3 (c): contrast between red/orange peppers and greenish peppers is increased), to exaggerate or trivialize, and certain cinematographic concepts such as calmness or stress.

2) By temporally varying style parameters based on the objects, the artist can maintain the aforementioned coherence and control, or modify the parameters, such as

- M. Kagaya, W. Brendel, Q. Deng, S. Todorovic, and E. Zhang are with the School of Electrical Engineering and Computer Science, Oregon State University, Corvallis, OR, 97331.

E-mail: kagaya|brendel|dengqi|sinisa|zhange@eecs.oregonstate.edu.

- T. Kestersonis with the New Media Communications, Oregon State University, Corvallis, OR 97331.

E-mail: kesterson@oregonstate.edu.

- Patrick J. Neill is with NVidia, 2701 San Tomas Expressway Santa Clara, CA 95050.Email: pneill@nvidia.com. achieving a rack focus effect (Fig. 9).

3) By allowing region-based brush stroke orientation design, the user has additional tools to achieve the aforementioned effects as well as to create illusions (Fig. 4).

Despite the potential benefits enabled by multi-style painting, there has been relatively little research in this area. Most existing work in image and video painting has focused on mimicking a single painting style where the style parameters such as brush stroke length, width, and opacity are constant over the whole spacetime domain [1], [2], [3], [4], [5], [6], [7], [8]. While there has been some work that can enable certain region-based non-photorealistic effects [9], [10], their focus is to typically on automatic stylization for some particular effect. Consequently, there is relatively little exploration on the artistic design of multi-style effects once the segmentation is available. Furthermore, there is little work in systematic design of brush orientations in video painting [11].

In this article, we address these issues by providing a painterly rendering framework for videos. In this framework, the video is first segmented into regions (objects or background) in temporally coherent fashion using an existing technique [12]. Next, the user defines style parameters and brush orientation for desired objects by specifying them in some keyframes. These user specification are then automatically propagated to the other frames as well as regions without any specifications through constrained optimization. Finally, the style parameters and stroke orientations will be used to generate the final rendering.

Our approach facilitates interactive artistic design and experiments once the segmentation becomes available. Style parameters can be specified in a small number of objects in some keyframes and automatically propagated to the whole video. In addition, as part of approach, we have developed 


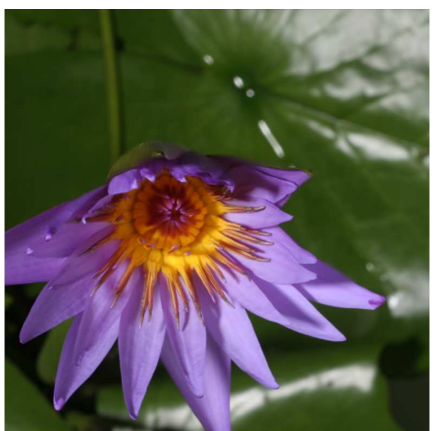

(a)

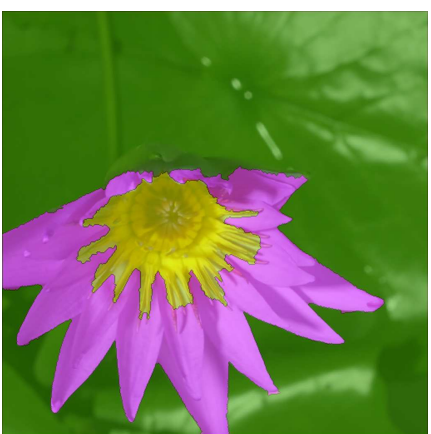

(b)

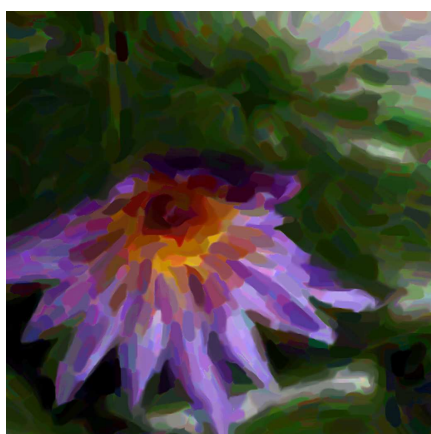

(c)

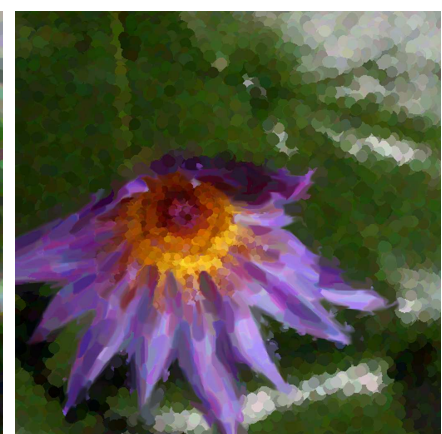

(d)

Fig. 1. Two stylized frames from a video showing a blooming flower: (a) the original frame, (b) the segmentation of the frame into three parts: petals, leaves, and stamens, (c) a single van Gogh style is applied to the entire frame, and (d) the same van Gogh style applied to the petals, whereas spatially varying Pointillist settings are applied to the leaves and stamens with different stroke diameters. The use of different styles, and spatially varying style parameters results in de-emphasizing the leaves, portraying the realistic fine granularity of the stamens, and enhancing overall contrast among the three regions. This is possible with the ability to produce temporally coherent segmentation.

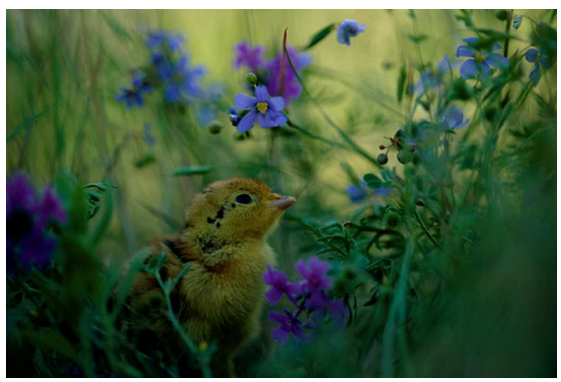

(a)

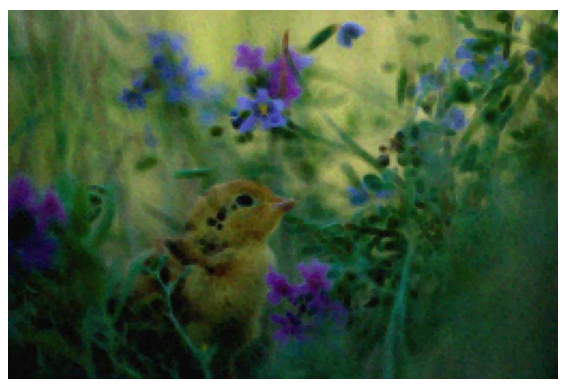

(b)

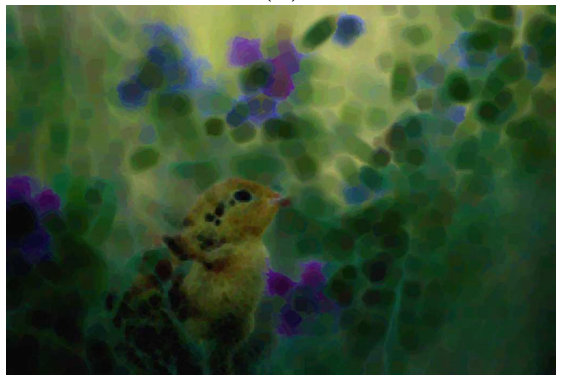

(d)

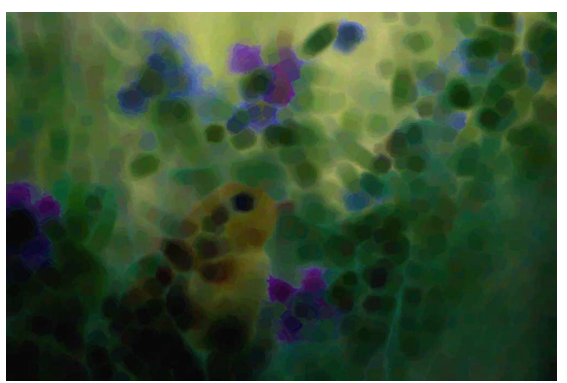

(c)

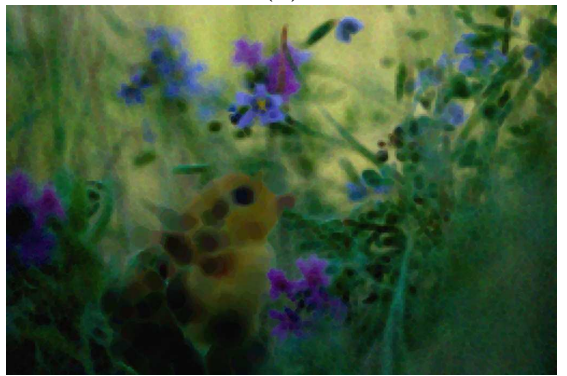

(e)

Fig. 2. An image of a bird (a) is painted in constant style parameter values (b-c) and spatially-varying values (d-e). The difference between (b) and (c) is the size of the strokes. In (b) details are preserved while in (c) the image is abstracted uniformly. With a binary segmentation (bird and background), the artist controls the amount of abstraction as well as the focus in the painting results (d: focus on the bird; e: emphasis given to the background flowers).

an image-based painterly rendering algorithm that can lead to more coherent results than existing geometry-based method in which curved brush strokes are explicitly defined. Moveover, we make use of the color of strokes to sort brush strokes, which has resulted in strokes with softer edges and partially alleviated the flickering issue in the video setting. Finally, we allow the user to easily design brush stroke orientations in a video, which, to the best of our knowledge, is the first of its kind. Note that the brush stroke orientations can be discontinuous across region boundaries, which is often desirable but has not been supported in past stroke orientation field design systems such as [13], [6], [11].
The rest of the article is organized as follows. Section 2 reviews related work. Section 3 describes the style and orientation design capabilities of our system and Section 4 explains our image-based multi-style renderer for images and videos. In Section 5 we describe how we generate a spatiotemporal video segmentation. We present results in Section 6 and summarize and discuss some future work in Section 7.

\section{Related Work}

We will review two areas that are most relevant to the contributions of this article: painterly rendering and flow visualization. 


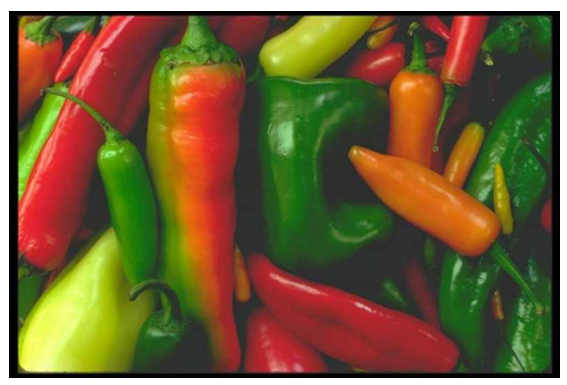

(a)

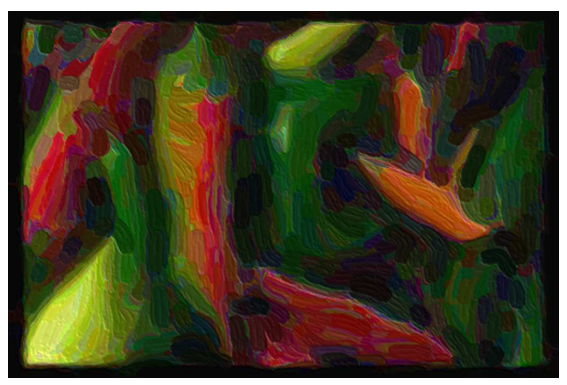

(b)

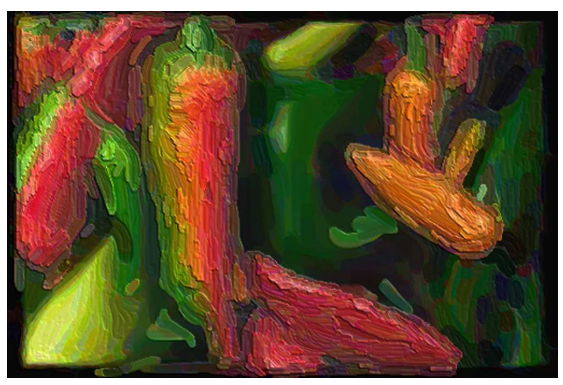

(c)

Fig. 3. This figure illustrates the control over the increase or decrease in contrast with spatially-varying style parameters. Given an image of peppers of various colors (a), two paintings are produced: (b) single-style, and (c) multi-style. Notice that the painting in (c) is obtained from (b) when increasing brightness and the amount of the detail to red and orange peppers only.

Painterly Rendering: Painterly rendering has been a wellresearched area in computer graphics. To review all the work is beyond the scope of this article. We here focus on the most relevant work.

Haeberli [1] introduces the idea of painterly rendering as a form of visual representation of objects. Litwinowicz [2] presents a system in which Impressionistic effects can be created for images and videos based on user specified stroke parameters. Hertzmann [13] describes an automatic algorithm that generates a number of styles of painting effects with various stroke sizes. A more physically realistic affect by adding height fields onto brush strokes which is used to add shading effect to the strokes [5]. Hertzmann [3] describes another painterly rendering method by formulating brush stroke placement as an energy optimization problem. Brush strokes can be added and removed as the optimization process iterates. In a rather different approach, Hertzmann et al. [14] augment images with painterly appearance using texture synthesis techniques.

Various techniques can be presented to produce video painting. Hertzmann and Perlin [7] extend the multi-layer approach of Hertzmann [13] to videos. In this approach, brush strokes from the previous frame will reused in the next frame after being moved according to the optical flow. Klein et al. [15] extend the notion of brush strokes to tubes in 3D spacetime, leading to more coherent but sometime undesirable appearances. Hays and Essa [6] provide a high-quality painterly renderer for videos by reusing the idea of optical flow and by restraining the speed of brush stroke rotation. DeCarlo and Santella propose a framework for abstracting a video [4], which is later used to produce video watercolorization [8]. In addition, Meier produces coherent rendering results for synthetic 3D scenes using a combination of geometry- and image-based techniques [16]. Cunzi et al. [17] address the "shower door" effect in 3D virtual walkthrough by moving the background in a plausible manner. These techniques assume a single set style parameters while our approach assumes space-time-varying styles parameters.

All of this work focuses on the rendering aspect of the painting. In contrast, we wish to provide a system that facilitates and supports the design and rendering process with varying style parameters. Hertzmann's work [13], [3] in providing level-of-detail control to the user is inspirational to this paper. However, we provide a more systematic approach that includes an effective segmentation tool and a user interface for varying style parameter design. Moreover, in this article we introduce the idea of creating a time-dependent tensor field to guide brush stroke orientations in a video. Past work [18], [11] applies tensor field design to image painting only. Based on our literature review, our system is the first that enables the time-dependent design capability.

Flow Visualization: In this article, we present a painterly renderer that is inspired by flow visualization techniques (Section 4). To review all related work in flow visualization is beyond the scope of this article, and we will only mention the most relevant work.

An efficient way of visualizing a vector field is by showing a set of streamlines, i.e., curves that are tangent to the vector field everywhere along their paths. Cabral and Leedom [19] present one such technique by performing line integral convolution (LIC) on an initial texture of white noise according to the vector field. For every pixel, they assign a value by extracting the streamline that contain the pixel and computing the average intensity of the initial texture along the streamline. The approach results in a high-quality continuous representation of the vector field. However, it is computationally expensive since it requires tracing a streamline for every pixel. Later, Stalling and Hege describe a faster way of creating LIC images by reducing the number of streamlines that need to be traced (FastLIC) [20]. Van Wijk [21] develops an interactive and high-quality image-based flow visualization technique (IBFV) for planar vector fields. IBFV enables interactive display of vector fields with the assistance of graphics hardware.

Zhang et al. [11] demonstrate that the edge field extracted from an image is a tensor field. They also point out that treating a tensor field as a vector field by simply removing the ambiguity in the orientation causes visual artifacts. Delmarcelle and Hesselink [22] propose to visualize 2D or 3D tensor fields with hyperstreamlines, which are curves that tangent to the major eigenvectors of the tensor fields everywhere along their 


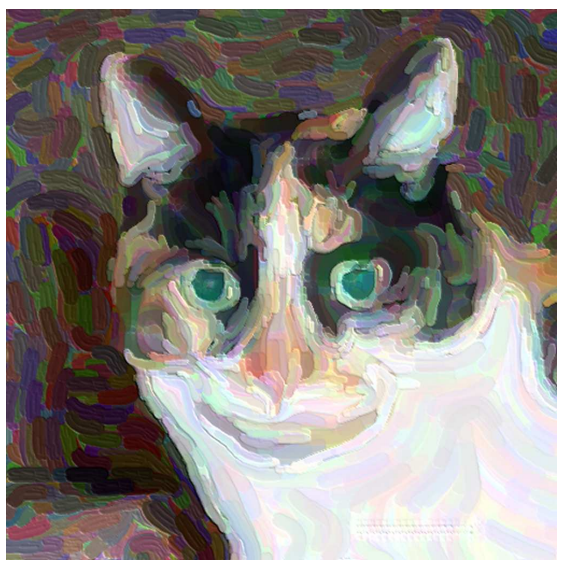

(a)

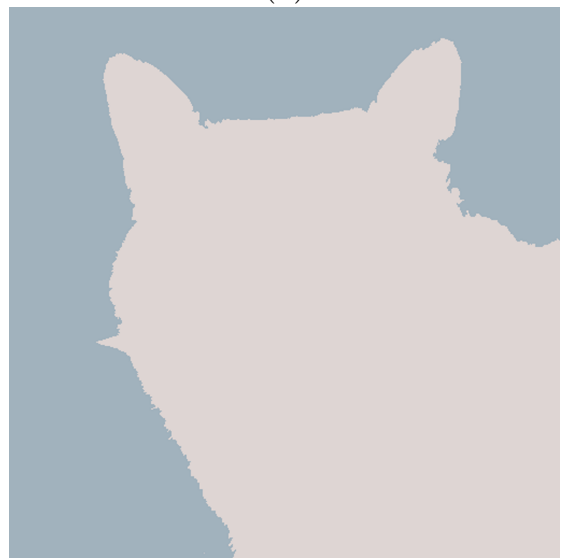

$(d)$

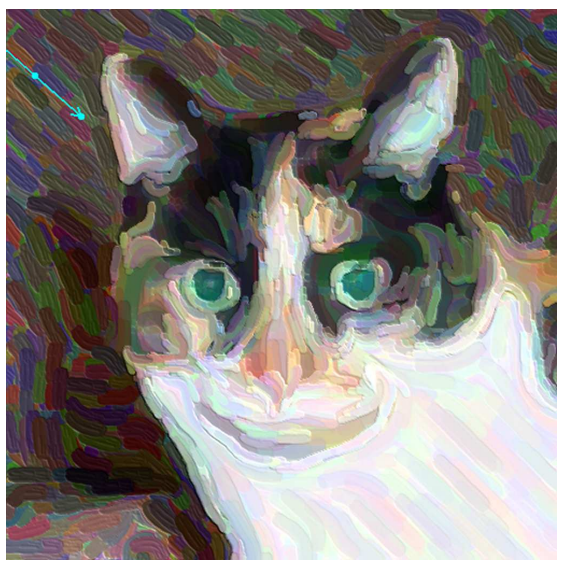

(b)

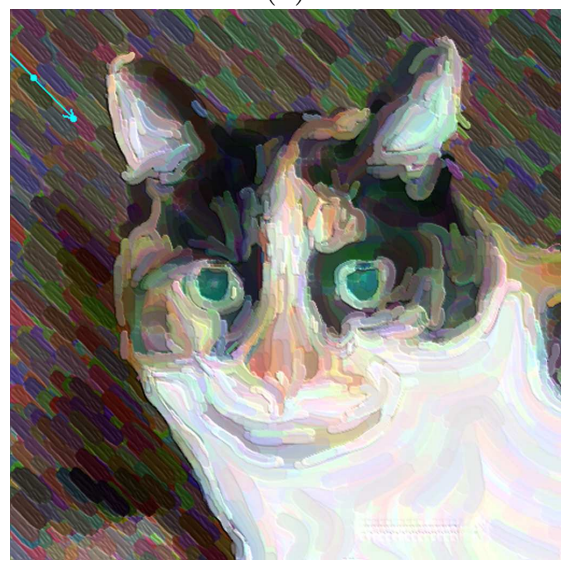

(e)

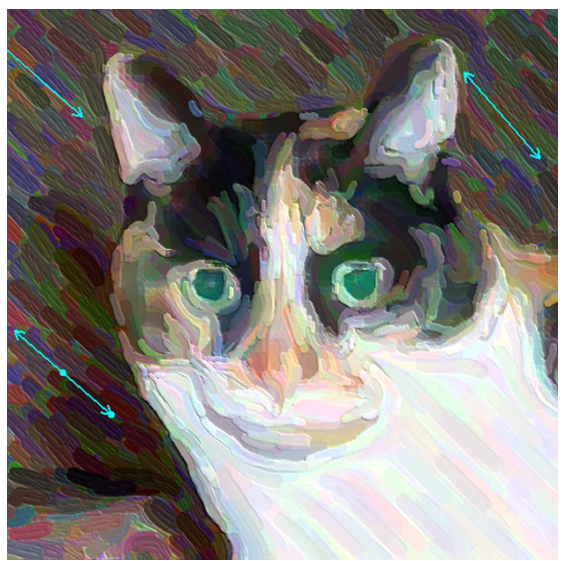

(c)

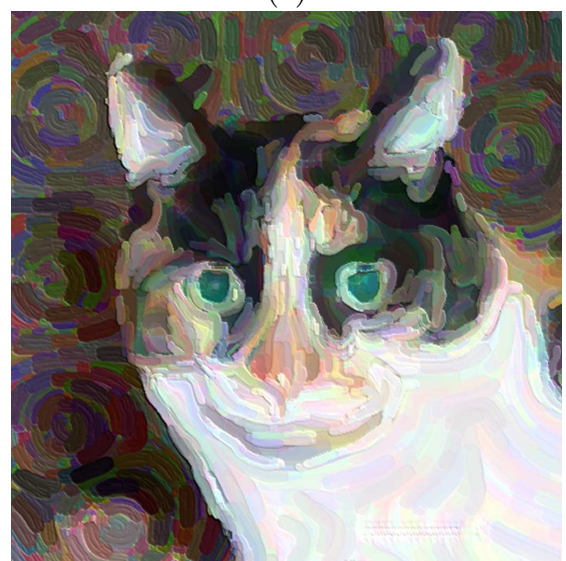

$(f)$

Fig. 4. This figure illustrates the importance of object-based stroke orientation design. Given an input of a cat, brush stroke orientation is based on the strong edges in the image (a). The user desires to assign a constant directional field for the background to achieve contrast between the background and the cat. Without segmentation, a single userspecified constraint (colored arrow in (b)) can only help achieve the artistic goal in a very local region (b). By adding more constraints in (c), the stroke orientations in the background start to conform. However, this has the side effect that these constraints also impact the orientation fields in the cat (white regions on her body). With the segmentation (d), the user can easily achieve the artistic goal with one constraint without modifying the stroke orientations inside the cat (e). Notice the stroke orientations in (e) is the same as in (a). In (f) hallucinational circular patterns are added to the background, again, without impacting the stroke orientations in the cat.

paths. Zheng and Pang [23] propose a tensor field visualization technique that they call HyperLIC. This method makes use of LIC to produce images that resemble visualizations based on hyperstreamlines. Zhang et al. [11] adapt the IBFV approach of van Wijk to tensor fields, which results in an interactive and high-quality tensor field visualization.

Our rendering pipeline adopts the approach Zhang et al. [11]. However, the application requirement is very different. We will provide details about our advection algorithm in more detail in Section 4.

\section{Style and Orientation Field Design}

Our multi-style rendering framework consists of three pipelines:

1) Video analysis and segmentation
2) Segmentation-enabled style and orientation design

3) Rendering

In the first stage, the input video $V$ is analyzed to compute the following information: the per-frame edge field $E$ and optical flow field $f$ as well as a temporally-coherent segmentation of $V$ into regions $R_{i}(1 \leq i \leq n)$ in the space-time domain.

In the second stage, the user specifies style parameters and designs stroke orientations based on the segmentation. This results in a number of scalar fields $s_{j}(1 \leq j \leq m)$, each of which corresponds to a style parameter such as stroke width or opacity, as well as a tensor field $T$ that guides brush stroke orientations in the video.

In the last step, an output video $V^{\prime}$ was produced by our painterly rendering algorithm that makes use of the segmentation, style parameters, optical flow, as well as the tensor field. 
The contributions of this work lie in the second and third stages, which we describe in detail in this section and Section 4 , respectively. In Section 5 we will mention existing techniques that we employ for video analysis, such as the segmentation method of Brendel and Todorovic [12].

In the second stage, the user can interactively specify desired style parameters as well as brush stroke orientations. These parameters will then be used in the final stage of rendering the output video (Section 4). The style parameters that our system supports include the following, most of which are inspired by work of Haeberli [1] and Hertzmann [13].

- Stroke size: describes the diameter of a stroke for Pointillism styles and the width of the stroke for other styles such as Impressionism, Expressionism, Van Gogh, and Colorist Wash.

- Stroke length: is desirable brush stroke length which allows long, curved brush strokes and can be used illustrate natural anisotropy in the images such as silhouettes.

- Stroke opacity: controls the amount of transparency or hardness in a brush stroke and is useful achieving styles such as the Colorist Wash.

- Color shift: describes changes in colors of all brush strokes in the video or a region. It consists of three parameters that describes the shift in Hue, Saturation, and Value, respectively. This parameter can be used to alter the overall tone of the image (Fig. 8).

- Color jittering: controls the maximal amount of jittering in the color of each brush stroke. When this parameter is zero, the color of each brush stroke is taken from the pixel in the input video corresponding to the seed location of the stroke. Having a non-zero value allows greater variation and thus contrast between neighboring brush strokes, which is desirable in Expressionism and the Van Gogh style [6].

- In-stroke texture height and density: allows a stroke to be textured, as in [5].

In addition to these art-driven parameters, we also support a number of algorithm-specific parameters such as:

- Number of layers: which allows layers of brush strokes of different sizes to be overlaid [13].

- Error threshold: is used to decide where on the canvas additional strokes are needed [13].

Our system assumes that style parameters are defined at each pixel.

The orientation of strokes is an important ingredient in conveying features with directions, such as the silhouette and motions of objects in the scene. Unlike style parameters, the orientation field is not a scalar field and contains directional information. We will discuss the design of scalar-valued style parameters and the orientation field in Sections 3.1 and 3.2, respectively.

Fig. 5 shows the interface of our system. The window consists of four parts: a canvas (upper-left), a control panel (right), a

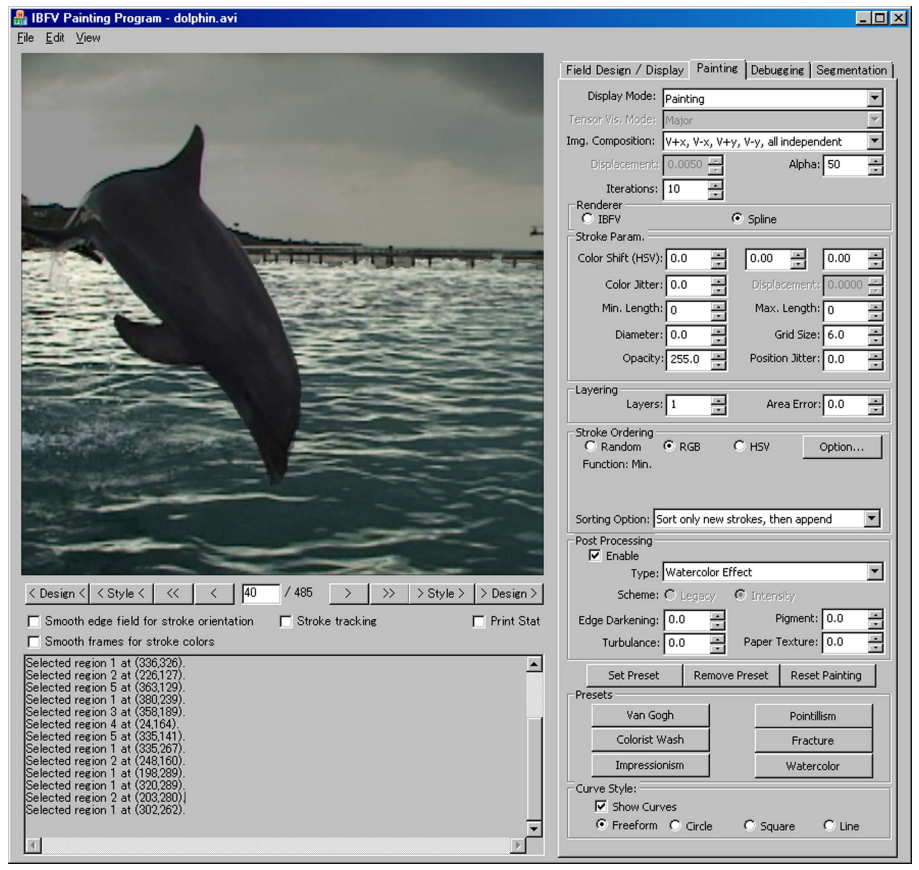

Fig. 5. This figure shows the user interface of our system.

frame browser (middle-left), and a system message box (lowerleft). The canvas can be used to show the input (image or video), segmentation, visualization of the orientation field, and the painterly result. When the input has been segmented, the user can select any region in the segmentation by clicking on a pixel belonging to that region. When this happens, other regions will be dimmed while the selected region remains its original colors. Clicking on a selected region will unselect it and undo the dimming of other regions. The user can then use the control panel to assign style parameters or design orientation fields for the selected region. For convenience, we have provided some default styles such as van Gogh, Colorist Wash, Impressionism, Pointillism, and Watercolor. The user can simply apply any of the these styles to the designed object by clicking the corresponding button. The style parameters can be modified at any moment and the corresponding result will be shown momentarily. In addition, the user can design brush stroke orientation using the control on the tab titled "Field Design / Display" (not shown in Fig. 5). Note that the controls for field design is a straightforward adaption from the tensor field design system of Zhang et al. [11]. The user can use the frame browser to jump to any frame or to the immediate previous or next keyframe for any given object, with either style parameters or stroke orientations. When this happens, any selected region will remain selected. Finally, the message box is used to provide feedback to the user with useful information. Next, we will describe the mathematical framework behind our design functionalities.

\subsection{Style Parameter Design and Propagation}

We will first discuss the design of spatially-varying style parameters in an image or a frame of a video. 
Given an input image $I$ and its segmentation $\mathscr{R}=$ $\left\{R_{1}, R_{2}, \ldots, R_{n}\right\}$, our system allows a user to select a region $R_{i}(1 \leq i \leq n)$ and associate a set of style parameters $\mathbb{S}=$ $\left\{s_{1}, s_{2}, \ldots, s_{m}\right\}$ to it. The style parameters are then assigned to every pixel in $R_{i}$. We refer to $R_{i}$ as a key region. Regions not receiving any specification are referred to as free regions. Our system automatically assigns values to free regions by propagating the parameters from the key regions through the following relaxation process. Let $R_{j}$ be a free region and $\mathbf{p}$ a pixel in $R_{j}$. Also, let $s_{k} \in \mathbb{S}$ be the $k$-th style parameter. Then

$$
s_{k}(\mathbf{p})=\sum_{r} \omega_{r} s_{k}\left(\mathbf{p}_{r}\right)
$$

where $\mathbf{p}_{r}$ 's are neighboring pixels of $\mathbf{p}$ in the image, and $\omega_{r} \geq 0$ is the weight of the $r$-th neighboring pixel satisfying $\sum_{r} \omega_{r}=1$. In our setting, we only consider four neighboring pixels and choose $\omega_{r}=\frac{1}{4}$. When $\mathbf{p}$ is on the boundary of the canvas we adjust the weights accordingly. The collection of these equations for pixels in the free regions gives rise to a system of discrete Laplace equations for which the parameter values of pixels in the key regions serve as boundary conditions. The discrete Laplace equation results in a sparse linear system which can be solved efficiently using a multi-grid Gauss-Seidel method [24].

Given a video $V$ and a temporally coherent video object segmentation $\mathscr{R}=R_{1}, R_{2}, \ldots, R_{n}$, our system allows the user to specify desired style parameters for a region $R_{i} \in \mathscr{R}$ in a set of keyframes $F_{i, j}: 1 \leq j \leq T(i)$ where $T(i)$ is the number of keyframes for region $R_{i}$. Note that $T(i)$ may vary for different regions $R_{i}$. Also, two regions $R_{i_{1}}$ and $R_{i_{2}}$ may not occur in the same keyframe. The specified values are automatically propagated to the rest of the video, i.e., to all pixels that were not given a style. Similar to the case of images, this can be achieved by using the Laplace equation as follows:

$$
s_{k}\left(\mathbf{p}^{t}\right)=\sum_{r} \omega_{r} s_{k}\left(\mathbf{p}_{r}\right)+\omega_{f} s_{k}\left(\mathscr{F}\left(\mathbf{p}^{t}\right)\right)+\omega_{b} s_{k}\left(\mathscr{F}^{-1}\left(\mathbf{p}^{t}\right)\right)
$$

where $\mathscr{F}\left(\mathbf{p}^{t}\right)$ and $\left.\mathscr{F}^{-1}\left(\mathbf{p}^{t}\right)\right)$ are the images of $\mathbf{p}^{t}$ under the forward and backward optical flows, respectively. The total weight $\sum_{r} \omega_{r}+\omega_{f}+\omega_{b}=1$. However, this formulation requires the equation be solved over the 3D domain of $M \times N \times L$ where $M, N, L$ are the number of rows, columns, and frames, respectively. While it is possible to solve this equation, doing so can reduce the interactivity that we wish to provide to the users. Instead of solving with the most general formulation given by Equation 2, we break the problem down into two sub-problems. First, given region $R_{i}$ and a set of corresponding keyframes, how do we estimate the style parameters of pixels in $R_{i}$ in a non-keyframe? Second, how do we estimate the style parameters for a pixel in region $R_{i}$ when there are no keyframes, i.e., $T(i)=0$ ? To address these questions, we consider the following two-step approach:

1) Given a region $R_{i}$ and a frame number $j$, we locate the closest previous and next keyframes involving $R_{i}$.
If both exist, then we assign style parameters to $R_{i}$ at frame $j$ by performing an interpolation between the style parameters of the two enveloping keyframes. The interpolation function $f(t)$ can be linear or nonlinear. Non-linear interpolation schemes allow the user to control the speed of transition between styles, which can be used to generate the effects such as calmness and stress. If only one exists, then we copy the style parameters from the keyframe. In the special case that $j$ is a keyframe for region $R_{i}$, no action is needed. This step ensures that the user specifications are continuously propagated from keyframes to the entire video.

2) We resolve style parameters of regions for which no keyframe was specified. Note that after the first step there is at least one region in each frame that has well defined style parameters. These values are simply propagated to the rest of the pixels in a frame by reusing Equation 1.

This two-step approach does not explicitly take into account the optical flow since the relaxation is done independently per frame. However, assuming that underlying video segmentation is sufficiently temporally coherent, the aforementioned approach approximates well the optimal solution.

\subsection{Stroke Orientation Field Design and Propagation}

Stroke orientation is an important parameter for providing artistic control. Zhang et al. [11] describe a tensor field design system for the purpose of guiding stroke orientation for image painting. The tensor field can be created from scratch or by modifying an existing field extracted from the image. User specifications are converted into basis tensor fields and summed. While this system has shown great promise in allowing stroke orientation control, it is inefficient for our purposes for two reasons. First, it is designed to work with only images and does not generalize to video in a straightforward fashion. Second, it assumes that the whole image is a single region and always generates a continuous tensor field. However, maintaining discontinuity in stroke orientations across object boundaries is often desirable. Despite these limitations, we feel that the idea of basis tensor fields is intuitive and effective. Consequently, we adapt this approach to construct a tensor field in a segmented video.

We first review relevant concepts of tensor fields and the idea of tensor field design by combining basis tensor fields. A second-order tensor $T$ is a $2 \times 2$ matrix $\left(\begin{array}{ll}t_{11} & t_{12} \\ t_{21} & t_{22}\end{array}\right) . T$ is symmetric if $t_{12}=t_{21}$ and traceless if $t_{11}+t_{22}=0$. In this article, we consider second-order symmetric, traceless tensors, referred to as tensors in the sequel, for simplicity. Such a tensor has the following form:

$$
T=\left(\begin{array}{rr}
u & v \\
v & -u
\end{array}\right)=\rho\left(\begin{array}{rr}
\cos \theta & \sin \theta \\
\sin \theta & -\cos \theta
\end{array}\right)
$$

where $\rho=\sqrt{t_{11}^{2}+t_{12}^{2}}$ and $\theta=\tan ^{-1}\left(\frac{t_{12}}{t_{11}}\right) . T$ is degenerate 
when $\rho=0$. A non-degenerate tensor has two eigenvalues $\pm \rho$ and two families of mutually perpendicular eigenvectors corresponding to the eigenvalues. Zhang et al. [11] use the eigenvectors corresponding to the eigenvalue $+\rho$ to guide the stroke orientations. We will follow this convention.

A tensor field is a continuous tensor-valued function. Useful tensor patterns for painterly rendering include regular patterns in which the eigenvector directions are the same everywhere and singular patterns such as wedges, trisectors, nodes and centers [11]. A basis tensor field corresponds to a pattern that can be specified by the following radial basis function:

$$
T_{i}(x, y)=e^{-d \sqrt{\left(x-x_{i}\right)^{2}+\left(y-y_{i}\right)^{2}}} M\left(x-x_{i}, y-y_{i}\right)
$$

in which $d$ is a decay constant, $\left(x_{i}, y_{i}\right)$ is the center of the basis function, and $M(s, t)$ is a function describing the tensor patterns. For example, a regular pattern can be characterized by a constant function $M=\left(\begin{array}{rr}u_{0} & v_{0} \\ v_{0} & -u_{0}\end{array}\right)$ while a wedge can be characterized by $M(s, t)=\left(\begin{array}{rr}s & t \\ t & -s\end{array}\right)$.

In the framework proposed in [11], a tensor field is generated by converting each user specification of a regular or singular pattern into a basis field and combining them. This framework has proven both fast and intuitive. In this article, we adopt the same framework and adapt it to segmented videos.

Given an image $I$ with segmentation $\mathscr{R}=R_{1}, R_{2}, \ldots, R_{n}$, a region $R_{i}$ is a key region with respect to orientation if the brush stroke orientation in $R_{i}$ is either specified by the user or automatically extracted from the image such as the edge field (Section 5). Otherwise, $R_{i}$ is a free region.

For a region $R_{i}$, the brush stroke orientation field is generated as follows: $P_{i}(\mathbf{p})=w_{i} E(\mathbf{p})+\left(1-w_{i}\right) \sum_{j} M_{i, j}(\mathbf{p})$, in which $E$ is the edge field extracted from the image, $M_{i, j}$ is the basis field corresponding to a user specification (design element [11]), and $w_{i} \in[0,1]$ is the weight constant assigned to the region. Notice that the region-based field design approach affords greater freedom in generating desired stroke orientations as the user need not be concerned with the impact of a user specification on pixels not in the intended region. In contrast, the single-region field design often requires the user to add additional specifications just to ensure that boundaries between regions are preserved by the field. Note that the center of a design element need not be inside the intended region. This is useful when an object is broken into several connected components such as the sun is partially occluded by the clouds (Fig. 8).

For regions where no user specification is given, our system assigns them values using the same framework in which style parameters are propagated (Section 3.1). Notice that in this case, we do not have a scalar field that represents some style parameter. Instead, we need to propagate directional information into free regions. Recall that the directional information is encoded by a tensor field, i.e., its major eigenvectors. Zhang et al. [11] have demonstrated that such propagation should be done by treating each of the four entries in the tensor field as an independent variable and solve the same Laplace Equation for each of them (Equation 1).

by solving a pair of Laplace equations similar to Equation 1 . Basically, we treat the entries in the tensor field as independent style parameters and reuse the multi-grid solved to propagate the tensor field to unknown regions. Note that for the type of tensors that we are concerned with, there are only two independent variables in Equation 3: $u=\rho \cos \theta$ and $v=$ $\rho \sin \theta$. Consequently, the amount of time to propagate stroke orientations is roughly twice of much for a style parameter.

Designing a tensor field for a video poses another challenge. In this setting, a set of constraints have been specified in different keyframes, and we wish to propagate them to the whole video. Similar to style parameters such as stroke length and opacity, we first propagate the basis tensor fields from keyframes to non-keyframes for regions where some user specifications exist. The only complication here is that we need the ability to update the center location $\left(x_{i}, y_{i}\right)$ and pattern function $M_{i, j}(x, y)$ of a user specification (Equation 4) from a keyframe to a nonkeyframe. This is necessary as the object such as a dolphin can jump and spin, and the user specification for one frame will not be able to achieve its original intention without accounting for the motion of the object. Note that we do not use optical flow for this purpose due to the noise and degeneracy often associated with its estimation. Instead, we obtain the center and orientation of an object by computing its best fitting ellipse through linear PCA analysis [25]. This allows us to track the motion of the object including translation (center movement), global rotation (axis rotation), and isotropic and anisotropic global scalings. We can now go through a non-keyframe and compute the tensor field values for pixels in key regions using updated center location and pattern functions. Next, values from the key regions in the frame are propagated free regions by the aforementioned tensor-valued relaxation process.

\section{Multi-Style Painterly Rendering}

Once all style parameters and stroke orientations have been populated to each pixel in every frame, we enter the third stage of our pipeline in which the video is processed according to the style parameters. We provide two algorithms.

\subsection{Explicit Stroke Method}

The first one is a straightforward adaptation from the renderer of Hertzmann [13] and Hays and Essa [6]. For images, this approach first places the brush stroke seeds (points) on a jittered 2D grid. Then every seed is traced along a directional field, which results in a streamline. The streamline is then fattened into a curved region (stroke) by a uniformly defined stroke width. Once canvas has been populated with these strokes, it is compared to a blurred version of the input image. Additional brush strokes with a smaller brush stroke width will be placed where the difference between the painting and the blurred image is larger than some threshold. This process 


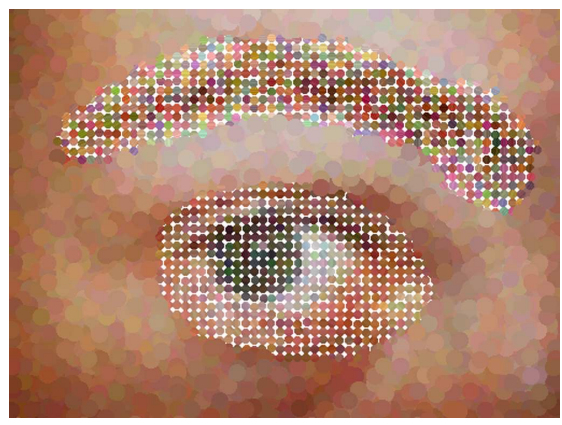

(a)

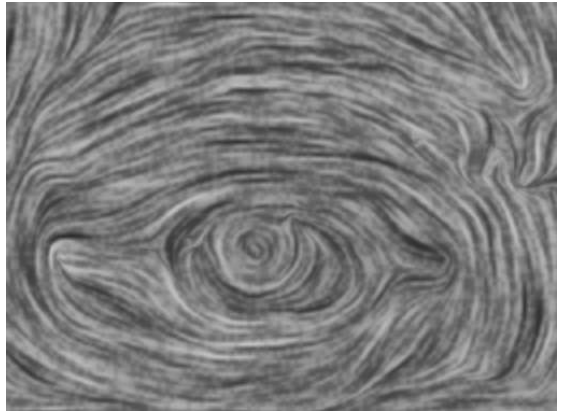

(b)

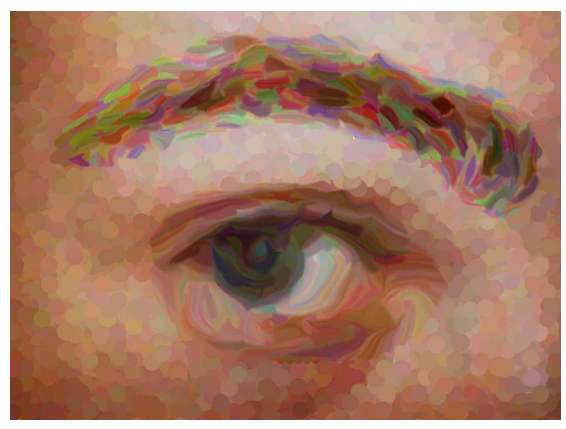

$(c)$

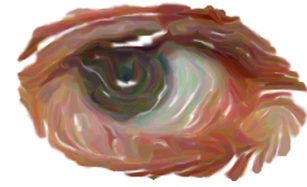

$(d)$

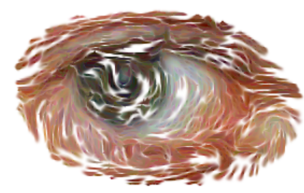

$(e)$

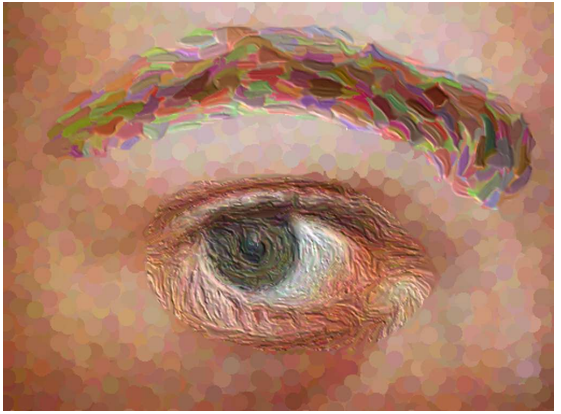

$(f)$

Fig. 6. An example workflow of our system: given the input image of an eye, the initial seeds are assembled in an image (a) which is advected according to the edge field shown in (b) to obtain the first-layer painting (c). Additional layers $(d, e)$ are generated in a similar fashion, which are then combined with the first layer to form the final multi-style rendering (f). The eye is rendered in a three-layer Impressionist style, the eyebrow in a one-layer van Gogh style, and the face in a one-layer Pointillist style.

then repeats. The resulting images can be further enhanced by embossing stroke textures to achieve a 3D effect [5]. It is straightforward to use this approach to support varying style parameters. The properties of a stroke are determined by the pixel for which it is centered. This way we do not have to recompute and re-evaluate the strokes during streamline tracing. Fig. 6 illustrates this process with the painting of an eye image using varying style parameters. For videos, we follow the approach of Hays and Essa [6] by using the optical flow to move the centers of strokes from the previous frame and by adding and removing strokes when necessary.

There are a number of complications. For example, the number of layers, an integer, is a style parameter in our system that can be propagated and interpolated. Yet, after the relaxation process this number must become a rational number. To overcome this problem, we snap the number of layers to the nearest integer while maintaining a continuously transitioned area error threshold. For a style that requires only one layer, we will set the corresponding area error threshold to the maximum number, which means no upper layer strokes are needed. Then, gradually lowering this number as one travels into multi-layer regions in which the area threshold is also lower, the number of upper layer strokes gradually increases.

Another problem is the brush stroke density, which leads to a strong sense of discontinuity. We do not address the density problem as it requires the handling of discontinuity in the number of strokes placed over the canvas. Instead, we assume a constant density for all styles. While this seems overly constraining, in practice we have found that the variety of styles our approach can simulate is not significantly affected by this limitation.

We have observed that this rendering technique seems to have a strong emphasis on individual strokes, especially for van Gogh and Impressionist styles. While this may be desired for image rendering, we have noticed much flickering due to the relative movement and visibility changes among overlapping strokes. Though we can use in-stroke opacity textures, it does not alleviate the problem due to the relatively small size of the strokes. To deal with this, we have developed a second renderer which we refer to as an implicit stroke method. For convenience, we will also refer to the renderer of Hertzmann [13] and Hays and Essa [6] as an explicit stroke method.

\subsection{Implicit Stroke Method}

Our implicit stroke method differs from the explicit stroke method mainly in one regard: stroke generation. To generate brush strokes on a layer, we first compute a set of seeds each of which is the starting point of a brush stroke on this layer. Next, every seed is drawn onto a temporary canvas $C_{0}$ as a colored disk where the color is from the pixel in the input $I$ or a blurred version of it. Finally, we advect the canvas as an image according to the stroke orientation field. The advection 


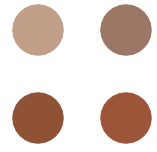

(a)

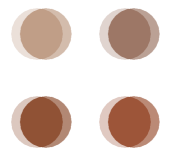

(b)

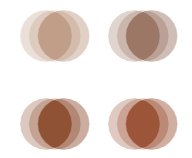

(c)
Fig. 7. This figure illustrates our process of advecting a source image (a) in the direction of an underlying vector field $V$. The image in (b) is obtained by warping (a) based on $V$ and composed with (a). Warping (b) and composing it with (a) results in (c).

is performed by iteratively warping the current canvas $C_{i}$ and compositing the result with the original canvas $C_{0}$ to obtain the new image $C_{i+1}$. Fig. 7 demonstrates this process. When composing the images at the pixel level, we face a challenge that the ID of the strokes are not well maintained during the warping of the canvas (as an image). Consequently, this can lead to inconsistent composition of pixels in overlapping strokes which in turn leads to color bleeding between them. To overcome this problem, we consider the following composite function $f$ for two images $A$ and $B$ :

$$
f(\mathbf{p})= \begin{cases}A(\mathbf{p}) & b_{B}(\mathbf{p})=1 \\ B(\mathbf{p}) & b_{A}(\mathbf{p})=1 \\ \min (A(\mathbf{p}), B(\mathbf{p})) & b_{A}(\mathbf{p})=b_{B}(\mathbf{p})=0\end{cases}
$$

where $b_{R}(\mathbf{p})$ is a binary-valued function that takes a value of 0 if the pixel $\mathbf{p}$ is covered by a stroke in image $R$. Otherwise, it is given a value of 1 . Basically, if $\mathbf{p}$ is not covered by any brush strokes in one of the images in composition, we use the color of $\mathbf{p}$ from the other image. This ensures consistent colors within a single stroke as it is being advected over the canvas. Note that this includes the case when $b_{A}(\mathbf{p})=b_{B}(\mathbf{p})=1$, i.e., the pixel is not covered by brush strokes in either image. In this case, the pixel belongs to background in both images and will remain so after composition.

When a pixel is covered in both images, i.e., there are at least two brush strokes that cover it, we choose the color which is smaller according to a total order on the space of colors. Given two colors $C_{1}\left(R_{1}, G_{1}, B_{1}\right)$ and $C_{2}\left(R_{2}, G_{2}, B_{2}\right)$, we choose $C_{1}$ if

1) $R_{1}^{2}+G_{1}^{2}+B_{1}^{2}<R_{2}^{2}+G_{2}^{2}+B_{2}^{2}$, or

2) $R_{1}^{2}+G_{1}^{2}+B_{1}^{2}=R_{2}^{2}+G_{2}^{2}+B_{2}^{2}$ and $G_{1}<G_{2}$, or

3) $R_{1}^{2}+G_{1}^{2}+B_{1}^{2}=R_{2}^{2}+G_{2}^{2}+B_{2}^{2}$ and $G_{1}=G_{2}$ and $R_{1}<R_{2}$, or

4) $R_{1}^{2}+G_{1}^{2}+B_{1}^{2}=R_{2}^{2}+G_{2}^{2}+B_{2}^{2}$ and $G_{1}=G_{2}$ and $R_{1}=R_{2}$ and $B_{1}<B_{2}$

Otherwise, we choose $C_{2}$. In practice, we have found less than $1 \%$ of total pixels ever require the second test. Notice that any total ordering can reduce bleeding between neighboring strokes. Finding the optimal ordering that achieves the best artistic looks is beyond the scope of this article and left for future exploration. The idea of a total color ordering has been used in adding security to images [26]. Notice our definition is slightly different from theirs.
While the implicit stroke method can also be accomplished through techniques such as line integral convolution [19] (LIC), we choose to adapt the technique of texture-based tensor field visualization technique of Zhang et al. [11], which is based on the image-based flow visualization (IBFV) technique of van Wijk [21].

The explicit and implicit methods can both support interactive design, with the explicit method being slightly faster (under 0.5 second per frame for one layer). On the other hand, the implicit method appears to focus less on individual strokes and thus is less sensitive to sudden change in strokes and more coherent in the video setting. All the example videos in this article were generated using the implicit method.

\section{Video analysis and Segmentation}

In this section we describe how we compute the optical flow and perform video segmentation. None of this work is novel as we reuse existing methods.

Edge Field and Optical Flow Estimation: An automatic video painting algorithm typically extracts the following information from the video: a directional field that orients brush strokes in each frame, and an optical flow field that moves brush strokes to follow the underlying objects that they depict [7], [6].

Our estimation of the directional field is based on [13], [11]. First, we estimate the image gradient vector field $G$ by using a Sobel filter. Next, we generate a second-order symmetric tensor field whose minor eigenvectors align with the image gradient vectors. The major eigenvectors of this tensor field are the directional field. Finally, we perform tensor field smoothing [11] in both space and time. This leads to more spatiotemporally coherent stroke orientations. Note that this directional field will be modified during the second phase of our framework: style and orientation design (Section 3.2). Moreover, it can be computed using other edge detection filters.

Optical flow estimation is done using the Lucas-Kanade method [27]. In practice, we compute both the forward and backward optical flow fields which allows us to transfer user-specification in both style and orientation design from keyframes to other frames (Section 3).

Spatiotemporal Video Segmentation: Obtaining spatiotemporal video segmentation is necessary for any region-based video processing operations, such as video matting [28], rotoscoping [29], and video tooning [9]. In our framework, it enables region-based style and orientation design. There are several approaches to achieving a spatiotemporal video segmentation [30], [31], [10], [32], [9]. For our purposes, any of these spatiotemporal video segmentation methods can be used. Specifically, we use the method presented in [12], because it does not require any models of, or prior knowledge about objects and their motions in the scene. Also, it is relatively fast and user friendly (see more detailed specifications in Section 6). 
We wish to emphasize that it is not the goal of this article to develop a new video segmentation method. Therefore, we will below only briefly review the segmentation method of [12] that we use in this paper, for completeness. The algorithm initially performs frame-by-frame 2D segmentation, and then tracks similar regions across the frames, such that the resulting tracks are locally smooth. Tracking is conducted by many-to-many matching of groups of spatially adjacent regions in one frame with groups of adjacent regions in the next frame. This partitions the spatiotemporal video volume into tubes that are coherent in space and time. Since region boundaries coincide with object boundaries, a cross section of the tubes and any video frame delineates all objects present in the frame. The extensive experimental evaluation of this method, presented in [12], suggests that the proposed approach compares favorably with the sate of the art.

\section{Results}

We have applied our system to a large number of example images or videos. Figs. 2, 3, and 4 provide examples in which an input image is processed using multi-style rendering to achieve various artistic goals. Next, we show frames from processed videos.

As the first example we show the power of spatially-varying styles with a video of a blooming flower that was segmented into three regions: stamens, petals, and leaves (Fig. 1 (b)). The stamens contain the highest-frequency details and the petals have strongest anisotropy. There is also motion in the leaves due to the movement of highlight (upper-right). Rendering the video using the same style parameters such as van Gogh (Fig. 1 (c)) often cannot adequately maintain the contrast between these characteristics. Using spatially-varying style parameters, the artist made the following style assignments: stamens (a Pointillist style), petals (van Gogh style), and leaves (a Pointillist style with a diameter twice as large as that for the stamens) (Fig. 1 (d)). With this setting, the detail in the stamens is maintained without sacrificing the contrast between the three regions.

The second example video shows a setting sun moving behind clouds (Fig. 8 (left)). The artist increased the contrast between the clouds and sun by gradually shifting the hue of the background (clouds and sky) from yellow to purplish blue through controlled color modification and by shifting the saturation of the strokes representing the sun toward pure colors (Fig. 8 (right)). This allows stroke movement in the dark sky region to become more visible, thus shifting the focus from the sun to the sky and clouds to reinforce that the night is coming. The design was inspired by van Gogh's Starry Night.

The third example highlights the benefits of time-varying styles in achieving an effect similar to a rack focus used in cinema. In a rack focus the lens focus is changed during a shot so that one object remains in focus while the other goes out of focus. This is used to direct viewer attention to different regions of the frame. In our example we are using variation in Pointillist brush size as a means of emphasizing or deemphasizing regions of an image. The video shows a woman, her child, and their interaction. The video is segmented into the woman, her hat and hair, the child, her hair, and the background. There are a number of motions in the video such as the woman smiling, pointing, and kissing the child as well as the child turning her head and smiling. Fig. 9 shows three frames from the output video in which the artist initially assigns a style to both faces with high details. Then the style is gradually varied to a low-detailed one for the child to reduce the attention on her as the mother points, turns her head, and smiles (A frame in which this has occurred is shown in Fig. 9 (left)). Later the style for the child transitions back to the original high-detail style as her mother kisses her. Meanwhile the style for the mother is switched to the same low-detail one used on the girl (Fig. 9 (middle)). Towards the end, the styles for both women are returned to the same original high-detailed one Fig. 9 (right). Notice the rack focus effect generated by varying style parameters over time.

In the next example we show temporal transition of stress to calmness with a video showing a dolphin repeatedly jumping out of water (Fig. 10). Starting with a sense of stress in which the artist shifts the stroke hues. This effect then smoothly transitions into a sense of calmness as the degree of color shifts is reduced.

In the last example we demonstrate the power of combining varying style parameters with stroke orientation design with the dolphin video. In Fig. 11 (left) the frame was rendered using the same style parameters for every object in the scene: the dolphin, the water, the sky, a mountain, and a bridge. The artist then assigned different style parameters to these objects (Fig. 11 (middle)) to add more details to the dolphin. The interaction between the dolphin and the water was further enhanced through stroke orientation design to create the illusion of ripples (Fig. 11 (right): where the dolphin touches the water).

The rendered clips corresponding to these images can be found in the supplementary video $^{1}$. The runtime of the employed video segmentation method [12] depends on the number of objects of interest and the frame size. For the dolphin video, it took about 30 seconds to process 100 frames without any user intervention. However, this process in slowed down by changes that the user may want to make in every frame. The design of style parameters and orientation fields depends on the number of objects, the number of keyframes, and the number of design changes to be made. For the flower video, where there are three regions, five keyframes, and the total design time was 230 seconds for 311 frames. Please note that the parameters for the supplementary video were designed by the artist on our team, who was a novice to our system and needed only a small period of time to be productive with our system - the period that is comparable to learning to use any other commercially available painterly rendering system. The design process is made easier with our system by allowing the user to start from

1. http://web.engr.orst.edu/ $\sim$ zhange/multistyle_painting.html 

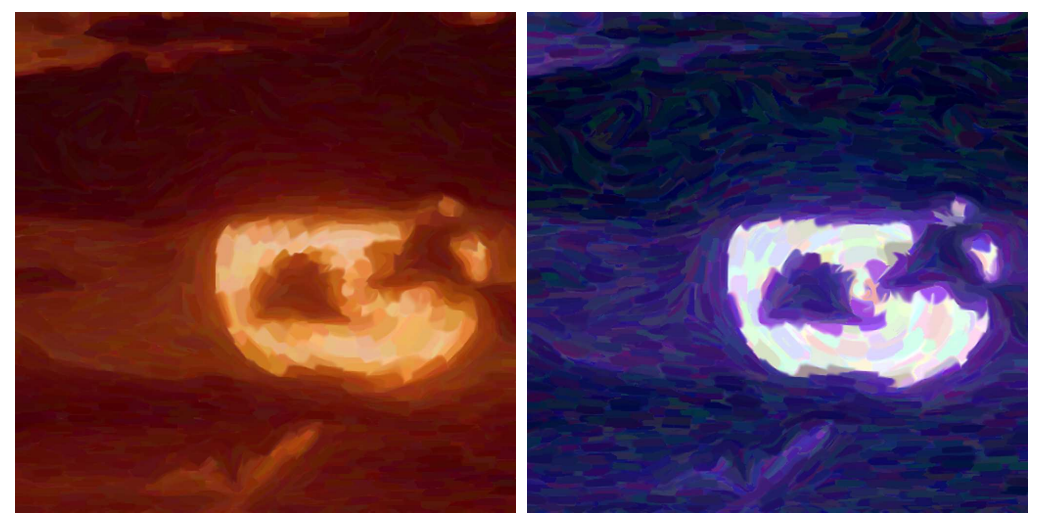

Fig. 8. Two frames from a video of sunset. Notice the change of hue in the background color allows the additional focus on the sky and clouds.
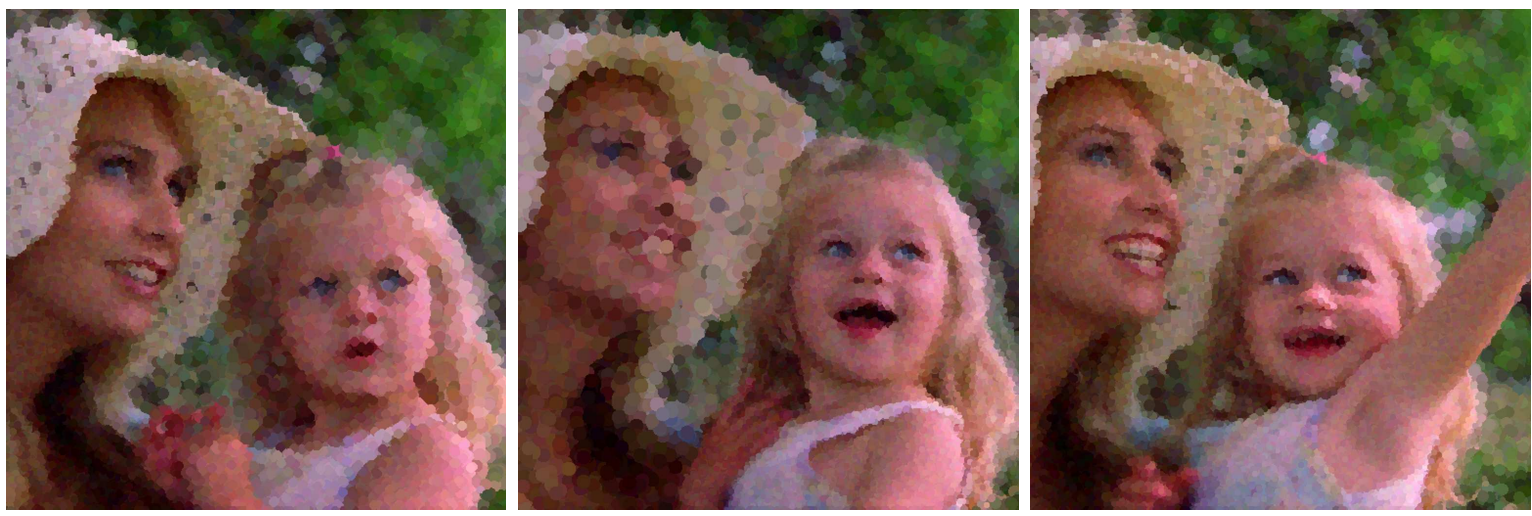

Fig. 9. Rack focus is shown on three frames from a video with space-time-varying stroke diameters: (left: Frame 161) both women in focus, (middle: Frame 429) mother in focus and girl out of focus, and (right: Frame 600) mother out of focus and girl coming into focus.

some existing styles and make a small number of adjustments. The orientation field design for the dolphin took approximately ten minutes. Furthermore, the rendering time for these videos is on the average of 2.5 seconds/frame. The segmentation was performed on a computer of $1 \mathrm{~GB}$ RAM and a $1.3 \mathrm{GHz}$ CPU. The design and rendering was done on a computer that has an NVIDIA 8800 GTX card with 512MB video memory and Pentium IV with a speed of $3.80 \mathrm{GHZ}$.

\section{Conclusion}

In this article, we describe a system for the design and painterly rendering of video with style parameters varying in space and time. Our system enables a wide range of artistic controls including brush stroke colors, widths, lengths, and opacity as well as brush stroke orientations. To our knowledge, the design of stroke orientation field in a temporally coherent fashion is the first of this kind. Our design tool is both interactive and intuitive. It can automatically propagate rendering parameters to the video thus reducing the amount of labor work. We provide two painterly renderers, explicit and implicit methods, by adapting existing painting methods and by applying flow visualization techniques, respectively. The implicit stroke method requires less focus on individual strokes and is thus less sensitive to sudden changes in stroke visibility. We have demonstrated the effectiveness of our system with several examples.

The efficiency of our system greatly depends on the quality of the employed segmentation method. For example, when there is semi-transparent object such as water in the dolphin video, we have noticed that it is difficult to always obtain a clean segmentation (See Fig. 12). As the dolphin jumps in and out of water, it is not always clear how to classify pixels that represent the part of dolphin underwater in a temporally coherent fashion. The vision researchers on this team plan to further investigate the issue as part of their future work.

Maintaining temporal coherence is still a great challenge. This is perhaps the most clear in the flower example, where there is stronger flickering effect toward the end when the flower has fully bloomed. Fig. 13 illustrates with three frames. Notice that there is little difference between the first frame in the sequence (left) and the immediate next frame (middle). However, when comparing the middle frame with the one in the right that is 10 frames after, it is quite clear that the order of neighboring strokes have changed in many part of the image. The relatively sudden visibility change between neighboring strokes is the source of the problem and requires further investigation. On the other hand, we note that the orientations of these strokes 

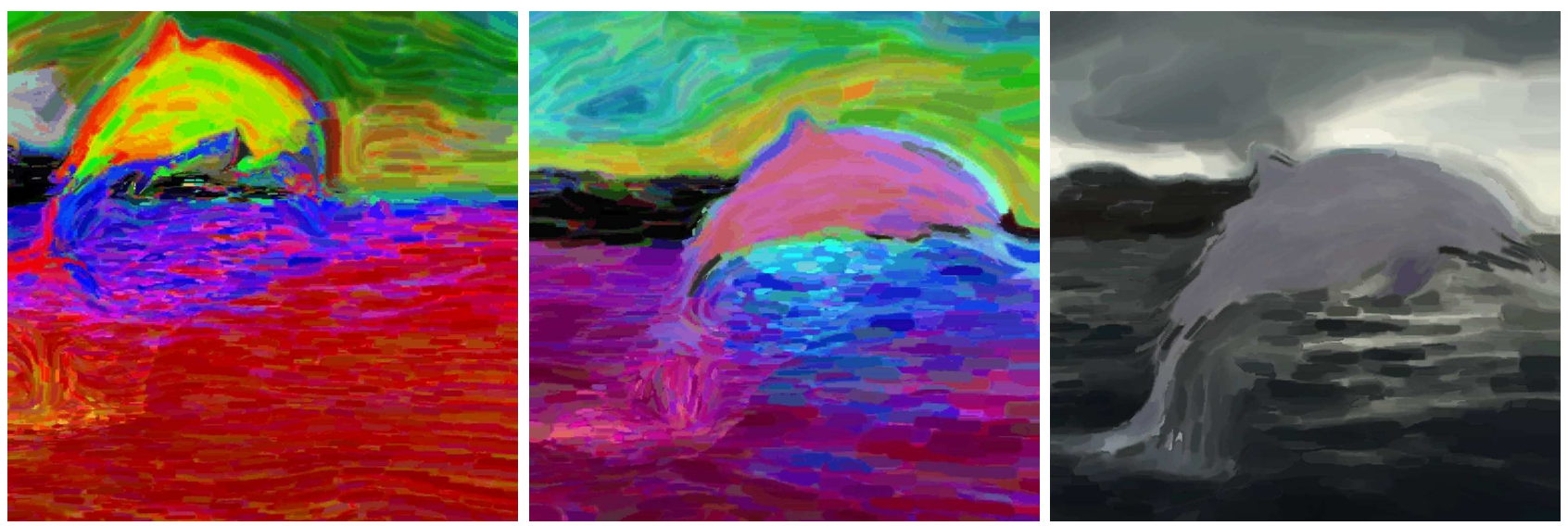

Fig. 10. Using space-time-varying style parameters we achieve a transition of a stress effect (left) to a calm effect (right). In (middle) the transition is shown.
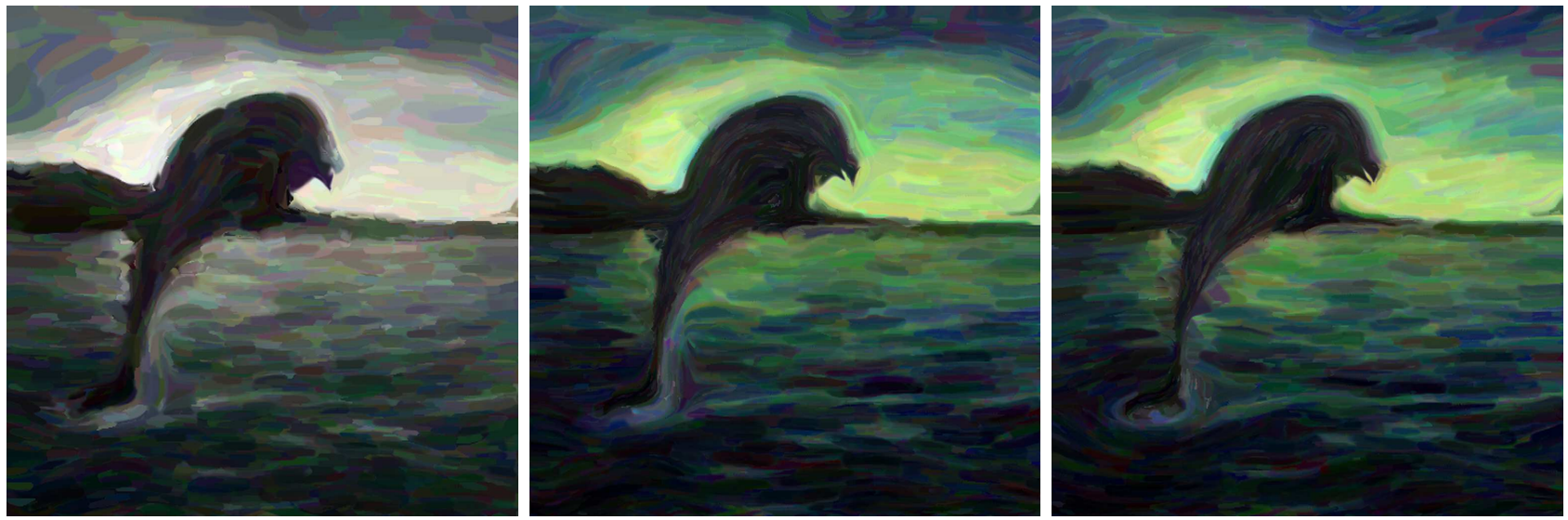

Fig. 11. Corresponding frames from three different renderings of a video of a swimming dolphin: (left) single-style, (middle) multiple styles, and (right) same as middle with additional design of stroke orientations. Notice that with control over stroke orientations (right), an illusion of rippling effect was added while the dolphin comes out of the water.

do not change significantly over time, indicating temporal coherence of our tensor fields.

There are a number of future research avenues for this work. First, we plan to investigate a more rigorous handling of time-dependent tensor field design. In particular, we wish to understand how to improve the quality of tensor field with explicit control over its smoothness and topology, such as singularities and bifurcations. We also plan to study how to edit the optical flow field using vector field design techniques. Second, the idea of using color orders to sort brush strokes is interesting, and we plan to pursue this direction in the near future. Finally, we are interested in means to propagate style parameters and orientation fields directly in the spacetime domain rather than the two-step approach we use in this paper. We believe that solving in 3D can lead to more smooth results.

\section{ACKNOWLEDGMENTS}

We would like thank all the reviewers whose constructive comments and suggestions have made this paper much stronger. We also wish to thank Artbeats.com for the videos used in this research as well as freefotos.com, pics4learning.com, US department of Agriculture, and Greg Turk for the source images. This work is sponsored by NSF grant CCF-0546881.

\section{REFERENCES}

[1] P. Haeberli, "Paint by numbers: abstract image representations," in $S I G$ GRAPH '90: Proceedings of the 17th annual conference on Computer graphics and interactive techniques. New York, NY, USA: ACM, 1990, pp. 207-214.

[2] P. Litwinowicz, "Processing images and video for an impressionist effect," in SIGGRAPH '97: Proceedings of the 24th annual conference on Computer graphics and interactive techniques. New York, NY, USA: ACM Press/Addison-Wesley Publishing Co., 1997, pp. 407-414.

[3] A. Hertzmann, "Paint by relaxation," in CGI '01: Computer Graphics International 2001. Washington, DC, USA: IEEE Computer Society, 2001, pp. 47-54.

[4] D. DeCarlo and A. Santella, "Stylization and abstraction of photographs," in SIGGRAPH '02: Proceedings of the 29th annual conference on Computer graphics and interactive techniques. New York, NY, USA: ACM, 2002, pp. 769-776.

[5] A. Hertzmann, "Fast paint texture," in NPAR '02: Proceedings of the 2nd international symposium on Non-photorealistic animation and rendering. New York, NY, USA: ACM, 2002, pp. 91-97. 

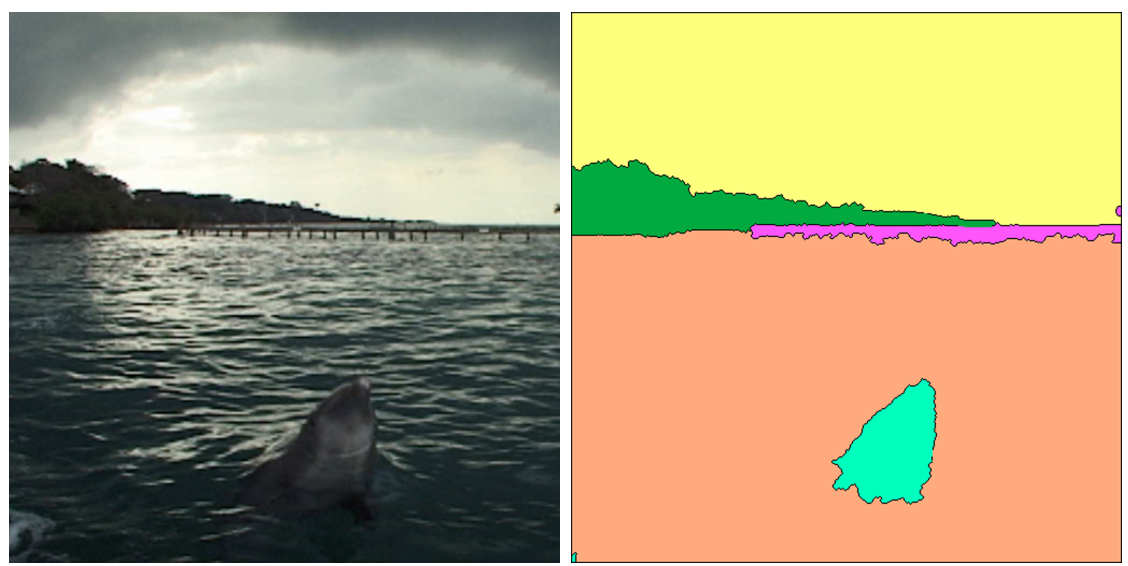

Fig. 12. A frame from the dolphin video along with its segmentation. Notice that part of the dolphin is underwater but visible due to the transparency of water. This makes it more challenging to obtain a coherent segmentation.
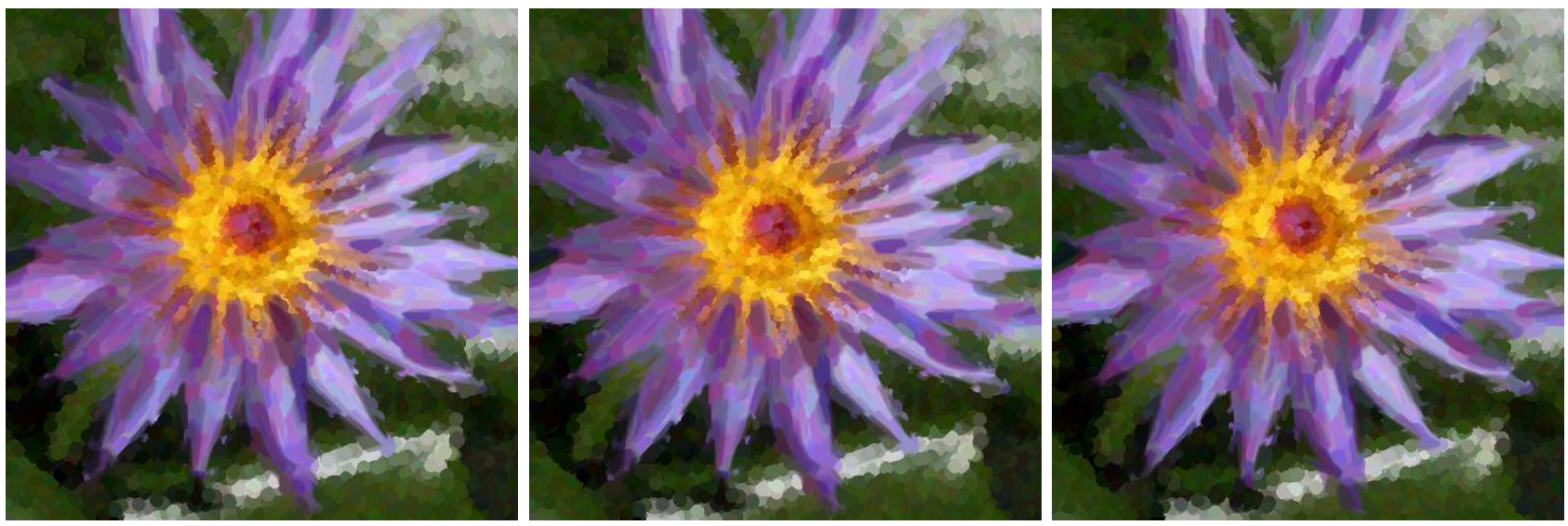

Fig. 13. An illustration of the flickering effect with the flower example. The middle frame is one frame after the left while the right image is ten frames after the middle. Notice that there is relatively change in stroke visibility between the left and middle frames, but significant changes between the middle and right frames.

[6] J. Hays and I. Essa, "Image and video based painterly animation," in NPAR '04: Proceedings of the 3rd international symposium on Nonphotorealistic animation and rendering. New York, NY, USA: ACM, 2004, pp. 113-120.

[7] A. Hertzmann and K. Perlin, "Painterly rendering for video and interaction," in NPAR '00: Proceedings of the 1st international symposium on Non-photorealistic animation and rendering. New York, NY, USA: ACM, 2000, pp. 7-12.

[8] A. Bousseau, F. Neyret, J. Thollot, and D. Salesin, "Video watercolorization using bidirectional texture advection," in SIGGRAPH '07: ACM SIGGRAPH 2007 papers. New York, NY, USA: ACM, 2007, p. 104.

[9] J. Wang, Y. Xu, H.-Y. Shum, and M. F. Cohen, "Video tooning," in SIGGRAPH '04: ACM SIGGRAPH 2004 Papers. New York, NY, USA: ACM, 2004, pp. 574-583.

[10] J. P. Collomosse, D. Rowntree, and P. M. Hall, "Stroke surfaces: Temporally coherent artistic animations from video," IEEE Trans. Visualization Computer Graphics, vol. 11, no. 5, pp. 540-549, 2005.

[11] E. Zhang, J. Hays, and G. Turk, "Interactive tensor field design and visualization on surfaces," IEEE Transactions on Visualization and Computer Graphics, vol. 13, no. 1, pp. 94-107, 2007.

[12] W. Brendel and S. Todorovic, "Video object segmentation by tracking regions," in IEEE Int. Conf. Computer Vision, 2009, pp. 1-8.

[13] A. Hertzmann, "Painterly rendering with curved brush strokes of multiple sizes," in SIGGRAPH '98: Proceedings of the 25th annual conference on Computer graphics and interactive techniques. New York, NY, USA: ACM, 1998, pp. 453-460.

[14] A. Hertzmann, C. E. Jacobs, N. Oliver, B. Curless, and D. H. Salesin, "Image analogies," in SIGGRAPH '01: Proceedings of the 28th annual conference on Computer graphics and interactive techniques. New York, NY, USA: ACM, 2001, pp. 327-340.

[15] A. W. Klein, P.-P. J. Sloan, A. Finkelstein, and M. F. Cohen, "Stylized video cubes," in SCA '02: Proceedings of the 2002 ACM SIGGRAPH/Eurographics symposium on Computer animation. New York, NY, USA: ACM, 2002, pp. 15-22.

[16] B. J. Meier, "Painterly rendering for animation," in SIGGRAPH '96: Proceedings of the 23rd annual conference on Computer graphics and interactive techniques. New York, NY, USA: ACM, 1996, pp. 477-484.

[17] M. Cunzi, J. Thollot, S. Paris, G. Debunne, J.-D. Gascuel, and F. Durand, "Dynamic canvas for non-photorealistic walkthroughs," in Graphics Interface, 2003, pp. 121-130.

[18] E. Zhang, K. Mischaikow, and G. Turk, "Vector field design on surfaces," ACM Transactions on Graphics, vol. 25, no. 4, pp. 1294-1326, 2006.

[19] B. Cabral and L. C. Leedom, "Imaging vector fields using line integral convolution," in SIGGRAPH '93: Proceedings of the 20th annual conference on Computer graphics and interactive techniques. New York, NY, USA: ACM, 1993, pp. 263-270.

[20] D. Stalling and H.-C. Hege, "Fast and resolution independent line integral convolution," in SIGGRAPH '95: Proceedings of the 22nd 
annual conference on Computer graphics and interactive techniques. New York, NY, USA: ACM, 1995, pp. 249-256.

[21] J. J. van Wijk, "Image based flow visualization," in SIGGRAPH '02: Proceedings of the 29th annual conference on Computer graphics and interactive techniques. New York, NY, USA: ACM, 2002, pp. 745-754.

[22] T. Delmarcelle and L. Hesselink, "Visualizing Second-order Tensor Fields with Hyperstream lines," IEEE Computer Graphics and Applications, vol. 13, no. 4, pp. 25-33, July 1993.

[23] X. Zheng and A. Pang, "Hyperlic," Proceeding IEEE Visualization, pp. 249-256, 2003.

[24] W. H. Press, S. A. Teukolsky, W. T. Vetterling, and B. P. Flannery, Numerical Recipes in C: The Art of Scientific Computing. New York, NY, USA: Cambridge University Press, 1992.

[25] N. J. Foster and A. C. Sanderson, "Determining object orientation using ellipse fitting," in IEEE Int. Conf. Computer Vision, 2009.

[26] C.-H. Tzeng, Z.-F. Yang, and W.-H. Tsai, "Adaptive data hiding in palette images by color ordering and mapping with security protection," IEEE Transactions on Communications, vol. 52, no. 5, pp. 791-800, 2004.

[27] B. D. Lucas and T. Kanade, "An iterative image registration technique with an application to stereo vision," in IJCAI, 1981, pp. 674-679.

[28] Y.-Y. Chuang, A. Agarwala, B. Curless, D. H. Salesin, and R. Szeliski, "Video matting of complex scenes," in SIGGRAPH '02: Proceedings of the 29th annual conference on Computer graphics and interactive techniques. New York, NY, USA: ACM, 2002, pp. 243-248.

[29] A. Agarwala, A. Hertzmann, D. H. Salesin, and S. M. Seitz, "Keyframebased tracking for rotoscoping and animation," in SIGGRAPH '04: ACM SIGGRAPH 2004 Papers. New York, NY, USA: ACM, 2004, pp. 584591.

[30] R. Megret and D. DeMenthon, "A survey of spatio-temporal grouping techniques," in Univ. Maryland Technical Report CS-TR-4403, 2002.

[31] A. Agarwala, "Snaketoonz: a semi-automatic approach to creating cel animation from video," in NPAR '02: Proceedings of the 2nd international symposium on Non-photorealistic animation and rendering. New York, NY, USA: ACM, 2002, pp. 139-148.

[32] Y. Park and K. Yoon, "Painterly animation using motion maps," Graph. Models, vol. 70, no. 1-2, pp. 1-15, 2008.

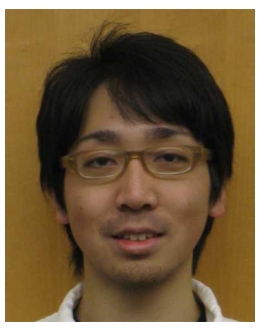

Mizuki Kagaya received the Bachelor's degree from Oregon State University, and he is currently continuing on study for the Master's degree in Computer Science at Oregon State University. $\mathrm{He}$ is working with Eugene Zhang, and his interested area in computer graphics research includes painterly rendering and geometric modeling.

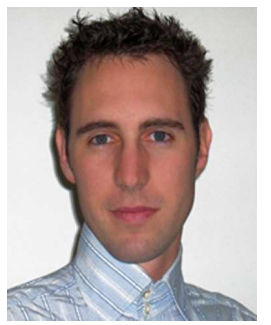

William Brendel received his Engineering Master at CPE Lyon, France in 2005. He worked for Infineon Technologies in Munich, Germany between 2003-2004 and for Laika Studios-House in Portland, OR in 2007. Currently William Brendel is a PhD student in the School of EECS at the Oregon State University. His research mostly focuses on graphics, computer vision and machine learning problems, including 3D reconstruction, feature detectors and descriptors, texture gradients, and spatiotemporal video segmentation.

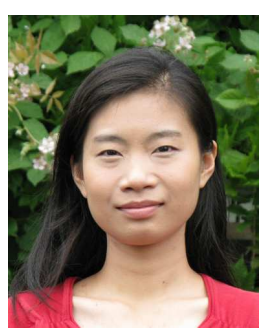

Qingqing Deng received the Bachelor's degree and Master's degree in Computer Science from Chongqing University, China in 2004 and 2007 respectively. She is currently working toward a Ph.D. degree in Computer Science at Oregon State University. She is working with Eugene Zhang on non-photorealistic rendering and vector field visualization.

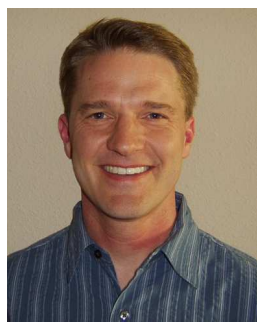

Todd Kesterson has worked professionally in the field of computer animation since 1990 on a wide range of projects for advertising, entertainment, and educational purposes. His work has been featured at SIGGRAPH, at Sea World (Florida and San Diego), and on National Geographic television. Kesterson holds an MS in Environmental Education from Southern Oregon University and an MFA in Interdisciplinary Arts from Goddard College in Vermont. He currently teaches courses in media aesthetics, conceptualization and production in the New Media Communications Program at Oregon State University.

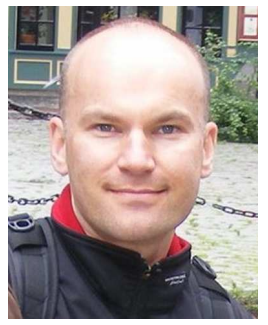

Sinisa Todorovic received his Ph.D. degree in electrical and computer engineering at the University of Florida in 2005. He was Postdoctoral Research Associate in the Beckman Institute at the University of Illinois Urbana-Champaign, between 2005-2008. Currently, Dr. Todorovic is Assistant Professor in the School of EECS at the Oregon State University. His research mostly focuses on computer vision and machine learning problems, including object recognition, texture analysis, and spatiotemporal video segmentation. He has published more than 30 journal and refereed conference papers. He is Associate Editor of Image and Vision Computing Journal, and Program Chair of 1st International Workshop on Stochastic Image Grammars (at CVPR 2009).

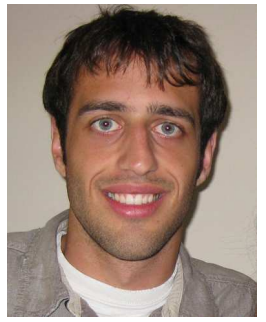

Patrick J. Neill received his BS and MS degrees in computer science, both from Oregon State University, in 2006 and 2008, respectively. His research interests include rendering and physical-based simulation. As a graduate student, he has worked on performing fluid simulation on interacting, deformable surfaces as well as painterly rendering of videos. He is working at NVidia.

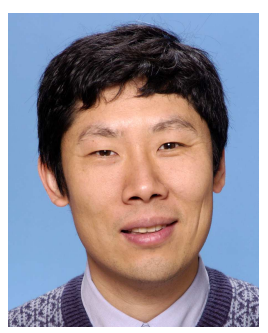

Eugene Zhang received the PhD degree in computer science in 2004 from Georgia Institute of Technology. He is currently an assistant professor at Oregon State University, where he is a member of the School of Electrical Engineering and Computer Science. His research interests include computer graphics, scientific visualization, and geometric modeling. He received an National Science Foundation (NSF) CAREER award in 2006. He is a member of the IEEE. 